ACCEPTED MANUSCRIPT

\title{
The influence of substrate roughness, patterning, curvature and compliance in peeling problems
}

To cite this article before publication: Lucas Brely et al 2017 Bioinspir. Biomim. in press https://doi.org/10.1088/1748-3190/aaa0e5

\section{Manuscript version: Accepted Manuscript}

Accepted Manuscript is "the version of the article accepted for publication including all changes made as a result of the peer review process, and which may also include the addition to the article by IOP Publishing of a header, an article ID, a cover sheet and/or an 'Accepted

Manuscript' watermark, but excluding any other editing, typesetting or other changes made by IOP Publishing and/or its licensors"

This Accepted Manuscript is @ 2017 IOP Publishing Ltd.

During the embargo period (the 12 month period from the publication of the Version of Record of this article), the Accepted Manuscript is fully protected by copyright and cannot be reused or reposted elsewhere.

As the Version of Record of this article is going to be / has been published on a subscription basis, this Accepted Manuscript is available for reuse under a CC BY-NC-ND 3.0 licence after the 12 month embargo period.

After the embargo period, everyone is permitted to use copy and redistribute this article for non-commercial purposes only, provided that they adhere to all the terms of the licence https://creativecommons.org/licences/by-nc-nd/3.0

Although reasonable endeavours have been taken to obtain all necessary permissions from third parties to include their copyrighted content within this article, their full citation and copyright line may not be present in this Accepted Manuscript version. Before using any content from this article, please refer to the Version of Record on IOPscience once published for full citation and copyright details, as permissions will likely be required. All third party content is fully copyright protected, unless specifically stated otherwise in the figure caption in the Version of Record.

View the article online for updates and enhancements. 
8 Trento, Italy.

9 (3) School of Engineering and Materials Science, Queen Mary University of London, Mile

15 Abstract

16

17

\section{The influence of substrate roughness, patterning, curvature and compliance in} peeling problems

\author{
Lucas Brely ${ }^{(1)}$, Federico Bosia ${ }^{(1)}$, and Nicola M. Pugno ${ }^{(2,3,4)^{*}}$
}

(1) Department of Physics and "Nanostructured Interfaces and Surfaces" InterDepartmental Centre, Università di Torino, Via P. Giuria 1, 10125, Torino (Italy).

(2) Laboratory of Bio-Inspired \& Graphene Nanomechanics, Department of Civil, Environmental and Mechanical Engineering, Università di Trento, via Mesiano, 77, I-38123 Trento, Italy.

End Road, London E1 4NS.

(4) Ket Lab, Edoardo Amaldi Foundation, Italian Space Agency, Via del Politecnico snc, 00133 Rome, Italy.

(*) Corresponding author: nicola.pugno@unitn.it

4

\section{Abstract}

Biological adhesion, in particular the mechanisms by which animals and plants "stick" to surfaces has been widely studied in recent years, and some of the structural principles have been successfully applied to bioinspired adhesives. However, modelling of adhesion such as in single or multiple peeling theories, have in most cases been limited to ideal cases and due consideration of the role of substrate geometry and mechanical properties has been limited. In this paper, we 1 propose a numerical model to evaluate these effects, including substrate roughness, patterning, 
1 curvature and deformability. The approach is validated by comparing its predictions with

2 classical thin film peeling theoretical model results, and then used to predict effects of substrate

3 properties. Results can provide deeper insight in experimentally obtained results and the

4 developed model a useful tool to design and optimize artificial adhesives with tailor made

5 characteristics.

6 Keywords:

7 Biological adhesion, thin film peeling, bioinspired adhesives, numerical modelling.

8 


\section{Introduction}

2 In recent years, there has been growing interest in the study of biological systems displaying

3 architectures that are optimized for specific functions such as structural resilience, protection,

4 locomotion, self-cleaning or self-repair [1] [2] [3]. The observation and mimicry of natural

5 systems has therefore led to the emergence of novel smart strategies to improve artificial system

6 functionalities [4]. This applies to the field of biological adhesion, which also studies the way in

7 which animals like beetles, spiders or geckos achieve optimized adhesion control by means of

8 arrays of tape-like micro- or nano-contacts organized in a hierarchical manner, especially in

9 heavier animals [5]. It has been demonstrated that these contact units can be modelled as

10 delaminating (or "peeling") thin films, rather than punch-like units [6], and that structures such

11 as gecko, spider or insect pads can be described using models based on this description of the

12 contacts [7] [8]. One of the key features of analytical tape peeling models, such as the Rivlin

13 model [9] or the Kendall model [10], is that the peeling force, i.e. the force necessary to initiate

14 detachment of the adhesive structure, strongly depends on the angle between the applied force

15 and the substrate, i.e. the "peeling angle". In addition to the observed contact geometries, one of

16 the main arguments for treating animal attachment as ensembles of delaminating tapes is that the

17 angle dependence on the detachment force has been experimentally observed in all species

18 displaying "hairy" contacts, both at micro and macro scale [6]. In addition to the geometry of the

19 tape unit and the peeling angle, the adhesive energy at the interface between the contact units and

20 the substrate contributes to determining the peeling force. This adhesive energy is mainly due to

21 van der Waals interactions [11] and to capillarity [12], but in the cited contact models, all

22 substrate and interface properties are condensed into a single adhesive energy term. For a given

23 loading scenario and tape geometrical/mechanical properties, the adhesive energy varies as a 
1 function of the material and the surface profile of the substrate [13] [14]. In turn, the peeling

2 force derived in analytical peeling models is related to the variation of the difference between

3 adhesive energy and total potential energy, which describes the micro scale physics of interface

4 failure.

5 With current progress in mimicking natural adhesives, the understanding of peèling mechanisms

6 and the derivation of adhesion optimization criteria become essential. To design optimal

7 solutions, adequate numerical tools taking account of all mechanical mechanisms are required,

8 and thus reliable models need to be developed. One of the main drawbacks of current models and

9 of Kendall's formulation in particular, is that they do not account for the dependence of the

10 adhesive force on substrate properties, i.e. the substrate is considered perfectly flat, perfectly

11 smooth and infinitely rigid. However, outstanding adhesive properties of animal attachment

12 systems also appear in the presence of variable substrate compliance, roughness, or of structured

13 surfaces [15]. These essential features should therefore also be included in models, especially in

14 the case of soft substrate materials.

15 Persson et al. [16] [17] presented a theoretical study on the influence of surface roughness

16 occurring at various size scales on the adhesive properties of biological adhesives, showing that a

17 hierarchical fibrillar structure is essential in guaranteeing sufficient adaptability to the surface.

18 Peng et al. proposed an analytical model to describe the effect of roughness on an elastic

19 nanofilm, linking its adhesion to the "wavelength" of the roughness profile [18]. Huber et al. [19]

20 and Cañas et al. [20] presented experimental studies on the effect of surface roughness on

21 artificial adhesives with spatula- or mushroom-shaped tips, including qualitative analysis of the

22 assumed mechanisms for adhesion enhancement for varying asperity size scales. The issue of 23 roughness as a cause for partial contact was addressed in [21], where the JKR theory was 
1 extended to account for energy released during rippling. A review of these and other modelling

2 approaches related to the influence of toughness are provided in [22]. However, in these works,

3 the effect of roughness or surface patterning on adhesive strength is not explicitly correlated to

4 the dimension of the process zone, i.e. the size scale at which stress concentrations occur in

5 peeling processes.

6 In this paper, we adopt a different approach to address the issue of surface roughness and

7 patterning, adding analysis of the influence of substrate curvature and compliance, with the

8 objective of better understanding the role of the substrate on the peeling behaviour of adhesives.

9 We therefore present a novel computational approach to model the so-called process zone of

10 peeling, which is the area where the interface between adhesive and substrate is subject to

11 stresses, leading to failure of adhesive bonds and therefore to detachment. The model is validated

12 with results from classical peeling theories and seyeral aspects of the interface/substrate are

13 discussed. 


\section{Analytical modelling of the process zone}

2 We consider a problem of dry adhesion of a contact unit on a substrate. The mechanics of its

3 detachment, illustrated in Figure 1.A, is analogous to the problem of a propagating crack front in

4 an adhesive interface. A classical energetic approach such as Kendall's model [10] provides a

5 macro-scale description of this mechanism, and is the reference model in the study of biological

6 adhesion. The progression of the peeling front becomes energetically favourable, and therefore

7 occurs if:

$$
\frac{\partial V}{\partial l_{d}}-\frac{\partial U_{e}}{\partial l_{d}}>\frac{\partial U_{s}}{\partial l_{d}}
$$

8 where $V$ is the work associated with the external load applied to the tape, $U_{e}$ the stored elastic

9 energy in the tape, $U_{s}$ the surface energy and $l_{d}$ the detached tape length, which increases as the

10 peeling proceeds. When delamination occurs, the dissipated energy per unit detached surface

11 area, indicated in Linear Elastic Fracture Mechanics (LEFM) as the strain energy release rate

12 [23], equals the adhesive energy $G$ per unit area of the interface:

$$
G=\frac{1}{w}\left(\frac{\partial U_{s}}{\partial l_{d}}\right)
$$

13 where $w$ is the tape width. According to Kendall's theory, this corresponds to:

$$
G=\frac{1}{w}\left(\frac{\partial V}{\partial l_{d}}-\frac{\partial U_{e}}{\partial l_{d}}\right)=\frac{F}{w}(1-\cos \theta)+\frac{F^{2}}{2 E b w^{2}}
$$

14 where $F$ is the applied load at the detached tape end, $b$ the tape thickness, $E$ the tape elastic

15 modulus and $\theta$ the angle between the applied load and the substrate (Figure 1.A). The critical 16 peeling force $F_{C}$ therefore becomes: 


$$
F_{c}=E b w\left(\cos \theta-1+\sqrt{(1-\cos \theta)^{2}+\frac{2 G_{c}}{E b}}\right)
$$

2 Another approach in the study of adhesion and delamination is to determine the loading state of

3 the interface, assuming a thin adhesive layer bonding the tape and the substrate [24]. In this

4 formulation, a finite-length region exists in the vicinity of the propagating delamination front,

5 referred to as the process zone, in which deformations in both the tape and the interface take

6 place [25]. Considering a simple loading scenario where the applied external load is parallel to

7 the substrate at $\theta=0$, the force balance acting on the attached region, according to the shear lag

8 model [26], can be written as:

$$
\frac{\mathrm{d} \sigma_{T}}{\mathrm{~d} x}=\frac{\tau_{I}}{b}
$$

9 where $\mathrm{d} \sigma_{T}$ is the infinitesimal variation of the tape axial stress over an infinitesimal length $\mathrm{d} x$

10 and $\tau_{I}$ is the shear stress in the interface layer, which is assumed initially to be a linear function

11 of the axial displacement within the tape $u$ and the interfacial stiffness $K_{I}$ (in the tangential

12 direction):

$$
\tau_{I}=K_{I} u
$$

13 Introducing the tape strain $\epsilon_{T}=\mathrm{d} u / \mathrm{d} x=\sigma_{T} / E$, after differentiation of Eq. (5), we obtain:

$$
\frac{\mathrm{d}^{2} \sigma_{T}}{\mathrm{~d} x^{2}}=\frac{K_{I}}{b E} \sigma_{T}
$$

14 This equation is solved applying the boundary conditions $\sigma_{T}(x=0)=F /(b w)$ and supposing 15 that the attached length $l_{a}$ of the tape is sufficiently long for the tape axial stress to tend to zero at 16 its edge $\sigma_{T}\left(x \rightarrow-l_{a}\right)=0$. In this case, we obtain the following distribution of interfacial stress: 


$$
\tau_{I}(x)=\frac{F}{w} \sqrt{\frac{K_{I}}{b E}} \exp \left(\sqrt{\frac{K_{I}}{b E}} x\right)
$$

1 In correspondence with the peeling line at $x=0$, the interfacial stress is maximum and failure of

2 the adhesive bond occurs when its elastic energy reaches the critical value:

$$
G_{c}=\frac{F_{c}{ }^{2}}{2 E b w^{2}}=\frac{\tau_{I c}^{2}(x=0)}{2 K_{I}}
$$

3 This equation also provides the relation between interface stress distribution and detachment

4 force. The critical peeling force in Eq. (9) is in agreement with Kendall's equation in Eq. (4) for

$5 \theta=0$, showing the consistency of the approach with existing peeling theories. Notice that

6 previous works have investigated the influence of bending stiffness on the peeling behaviour of

7 thin films [27]. In the present paper, we consider this to be negligible, and tape thickness values

8 will be chosen accordingly.

9 When the peeling angle differs from 0 , the adhesive bonds experience both tangential and normal

10 loads, whose distributions influence the external force at which the system detaches. The

11 interface is therefore subject to a mixed-mode fracture mechanism with an opening mode (mode-

12 I) and a sliding mode (mode-II). For small peeling angles, the mode-II strain energy release rate

$13 G_{I I}$ can be approximated by solving Eq. (7) with the boundary condition $F(x=0)=F \cos \theta$,

14 leading to:

$$
G_{I I}=\frac{F^{2}}{2 E b w^{2}} \cos ^{2} \theta
$$

15 The mode-I strain energy release is estimated injecting Eq. (10) into Eq. (3) with $G=G_{I}+G_{I I}$, 16 leading to: 


$$
G_{I}=\frac{F^{2}}{2 E b w^{2}} \sin ^{2} \theta+\frac{F}{w}(1-\cos \theta)
$$

1

2

3

4

5 overall adhesive performance. The tape is thus discretized with a set of truss members of length

$6 \Delta x$ (the discretization length), undergoing axial load only. A traction vs. separation law, usually

7 referred to as a Cohesive Zone Model (CZM) [28], is introduced for each tape node to model the

8 interface between the tape and the substrate, i.e. a restoring reaction force is generated on the

9 tape nodes as a function of the deformations of the system. The relation between the tape axial

10 stress and strain is taken as linear elastic for simplicity, but geometrical nonlinearities can occur

11 in interfacial stresses due to the nature of the problem. As the applied force and its angle with

12 respect to the substrate increase, deformations, translations and rotations of the tape vary in the

13 process zone, as does the stored elastic energy of the adhesive interface bonds, which are

14 responsible for the macroscopic behaviour of the peeling system. To calculate the mechanical 15 equilibrium after the application of the force, a Newton-Raphson iterative scheme [29] is adopted

16 (details are given in Appendix A). A 2-D model is employed, assuming that the detached and 17 attached tape regions and the applied force are coplanar. The detached part of the tape is not 18 modelled, since the far-field external load can be directly applied in correspondence with the 19 peeling line at $x=0$. This is because the tape bending stiffness is neglected, so that the detached 20 part of the tape only transfers axial load without influencing the mechanical equilibrium of the 21 process zone. We introduce a linear interfacial traction vs. separation law as: 


$$
\frac{R}{w \Delta x}=K_{I} \delta_{I}
$$

1 where $R$ is the nodal reaction force and $\delta_{I}=\sqrt{u^{2}+v^{2}}$ is the separation of a tape node with

2 respect to its initial position in the plane, with $u$ and $v$ the components of the nodal displacement

3 along $x$ and $y$. Note that interface bonds experience axial load only (tangential and normal forces

4 are coupled). A schematization of the numerical model is shown in Figure 1.B.

5 In the following, tape and interface properties are chosen as: $w=1 \mathrm{~mm}, b=0.01 \mathrm{~mm}, K_{I}=10$

$6 \mathrm{MPa} \cdot \mathrm{mm}^{-1}, l_{a}=2 \mathrm{~mm}$, and an external load of $F=10 \mathrm{~N}$ is applied. The tape Young's modulus is

7 chosen as $E=100 \mathrm{MPa}$ since most biological fibrillar adhesives reach values above $1 \mathrm{GPa}$ [17]

8 [22] [30], but recent studies indicate that the modulus decreases towards the tips of the contacts

9 [31]. The corresponding tangential and normal stress distributions, calculated from the

10 displacement field at equilibrium as $\tau_{I}=K_{I} u$ and $\sigma_{I}=K_{I} v$, as a function of the peeling angle

11 are shown in Figure 2.A and 2B. These distributions highlight the fact that the angle dependency

12 is related to stress distributions within the process zone. At $\theta=0$, the normal stress is zero and

13 the shear stress distribution agrees with the analytical solution in Eq. (8) (shown in red). We note

14 that the normal interfacial stress is more concentrated at the peeling line compared to the shear

15 one, and is mainly responsible for the decrease in the peeling force as the peeling angle is

16 increased. In addition, no compressive normal stress is present ahead of the peeling front,

17 consistently with the fact that with the chosen parameters, the bending stiffness is negligible.

18 In the numerical model, the mode I and mode II strain energy release rates are computed from

19 the displacement field at the most critical adhesive bond (in correspondence with the peeling 20 line) as follows: 


$$
\begin{aligned}
& G_{I}=\frac{1}{2} K_{I} v^{2} \\
& G_{I I}=\frac{1}{2} K_{I} u^{2}
\end{aligned}
$$

1 The dependence of $G_{I}$ and $G_{I I}$ on the peeling angle $\theta$, calculated both analytically and

2 numerically, is shown in Figure 2.C. There is a good agreement between analytical and

3 numerical results, but for large peeling angles, rigid body motions of the tape and large adhesive

4 bond deformations within the process zone affect the interfacial stress distribution and lead to a 5 discrepancy between the two.

6 We now introduce a local energy-based failure criterion, i.e. a critical strain energy release rate $7 G_{C}$ for the interface bonds, beyond which detachment occurs, as:

8 The corresponding peeling force as a function of the peeling angle calculated numerically agrees 9 with the analytical solution of Eq. (3), as shown in Figure 3 for an adhesive energy $G_{c}=0.5$ $10 \mathrm{MPa} \cdot \mathrm{mm}$.

11 In nature, a wide variety of types of bonding between the tape-like contacts and the substrate has 12 been observed in biological systems. The van der Waals interaction responsible for the adhesion 13 of Geckos, the capillarity forces in insect attachment or glue-coated spider silks all have specific 14 interactions with the substrate. The traction-separation law is therefore expected to depend on the 15 type of interaction. In the case of a nonlinear force vs. separation relation of the interface bonds, 16 the peeling force is not affected by the type of nonlinearity as long as the total energy dissipated 17 by a specific bond during loading is invariant. This means that although the model has been 18 developéd for dry adhesion, wet adhesion can also be simulated, provided an appropriate 
1 adhesive term can be formulated. We thus consider different traction-separation laws (Reaction

2 force $R$ vs. elongation $\delta_{I}$ ) in the model:

3 - Linear elastic:

$$
\frac{R}{w \Delta x}=\left\{\begin{array}{cl}
K_{I} \delta_{I} & \text { for } \delta_{I}<\delta_{I}^{*} \\
0 & \text { for } \delta_{I}>\delta_{I}^{*}
\end{array}\right.
$$

4 - Perfectly elasto- plastic:

$$
\frac{R}{w \Delta x}= \begin{cases}K_{I} \delta_{I} & \text { for } \delta_{I}<\delta_{I}^{*} \\ K_{I} \delta_{I}^{*} & \text { for } \delta_{I}>\delta_{I}^{*} \\ 0 & \text { for } \delta_{I}>\delta_{I}^{* *}\end{cases}
$$

$5 \quad-$ Exponential:

$$
\frac{R}{w \Delta x}=\frac{R^{*}}{w \Delta x} \frac{\delta_{I}}{\delta_{I}^{*}} \exp \left(1-\frac{\delta_{I}}{\delta_{I}^{*}}\right)
$$

6 The input parameters for each of these laws must satisfy the following condition:

$$
\int_{0}^{\infty} \frac{R}{w \Delta x} \mathrm{~d} \delta_{I}=G_{c}
$$

7 When the peeling force is reached, the load distribution at the interface $\frac{R}{w \Delta x}$ vs $x$ changes

8 considerably as a function of the chosen traction-separation law, as shown in Figure 4.A (for $9 \quad \theta=\pi / 8$ and $\left.G_{c}=0.5 \mathrm{MPa} \cdot \mathrm{mm}\right)$.

10 The applied force versus force application point displacement $\eta$ obtained for a peeling test, i.e.

11 when the system is loaded until complete detachment of the tape, is shown in Figure 4.B for an 12 exponential traction-separation law and $\theta=\pi / 8$, highlighting an "elasto-plastic" behaviour.

13 When the applied load is below the peeling force (points $a$ and $b$ ), the elastic energy in the 14 interface increases as it deforms. Next, the crack front propagates at constant peeling force (point 
1 c) over the attached length of the tape. A small drop of the peeling force is observed (point $d$ ) when the crack front reaches the end of the attached region, before complete detachment. This is because the remaining attached part is not long enough for the axial load in the tape, and

5

6

\section{Influence of substrate geometrical features}

We now study the influence of several characteristics of the substrate on the peeling force using the previously introduced numerical model. For simplicity, the linear-traction-separation law is employed for the interface in simulations, together with the local energy-based delamination criterion.

\subsection{Substrate roughness and patterning}

An imperfect contact between the tape and the substrate, as a result of surface roughness, can be introduced by statistically assigning a variable adhesive energy $G_{C}^{*}$ to each adhesive bond. These energy values are extracted randomly from a uniform distribution $G_{C}^{*}=2 \cdot U\left(0, G_{C}\right)$, and from a normal distribution $G_{C}^{*}=\mathcal{N}\left(G_{C}, G_{C} / 10\right)$ with $G_{C}=1 \mathrm{MPa} . \mathrm{mm}$. The first of the two is considered since it allows to evaluate the effect of a large scatter in adhesive energy values. The second of the two is closer to the surface roughness distribution found in real materials. The simulation is performed for $\theta=0$ and $\theta=\pi / 4$. The corresponding force-displacement plot of the force application point $\eta$ is shown in Figure 5.A. The general behaviour of the system is similar to that for a smooth substrate shown in Figure 4.B. The two plots refer to different traction-separation laws, but this does not influence the overall behaviour, provided the integral in Eq. (19) is the same. The peeling force appears to be approximately constant in spite of the high variability in the local failure energies. It is interesting to note that the obtained peeling forces are $F_{c}(\theta=$ $230) \cong 10 \mathrm{~N}$ and $F_{c}(\theta=\pi / 4) \cong 7.41 \mathrm{~N}$, which are approximately those predicted by Eq. (4) 
1 considering the mean value $G_{c}=0.5 \mathrm{MPa} \cdot \mathrm{mm}$. The time variation of the load distribution is

2 shown in Figure 5.B in the case of a uniform distribution at $\theta=0$, and helps gain a better

3 understanding of the peeling force results. In the early stages of loading of the system, some

4 local detachment events occur ahead of the crack front, in correspondence with the weakest

5 adhesive bonds, and the load is then distributed on the surviving ones, driving the peeling force.

6 Another important effect related to the substrate morphological properties is the possible

7 presence of entire regions where contact is absent, such as in the case of patterned surfaces. To

8 investigate this, we simulated numerically the case of a periodic pattern schematically shown in

9 Figure 6.A, by alternating regions without and with bonding between the tape and the substrate,

10 respectively of length $\alpha$ and $\beta$. The results of the simulations for $\theta=\pi / 8$ and varying $\alpha$ and $\beta$

11 values are shown in Figure 6.B. Here, the maximum peeling force for the considered structure is

12 normalized with respect to the one predicted by Eq. (4) i.e. for full contact over the entire

13 substrate. When the length of the attached region $\alpha$ reaches the theoretical process zone length,

14 the normalized force tends to 1 , which means that the pattern does not affect the process zone

15 distribution. As $\beta$ decreases, the maximum peeling force is reduced due to an increase in the load

16 concentration within the process zone. The size of the gaps within the attached region also

17 affects the peeling force. As the length $\alpha$ increases, the detachment force decreases for a fixed $\beta$.

18 Simulations show that patterned substrate surfaces are detrimental to the peeling force, especially

19 when the size of gaps in the interface is large with respect to the process zone length. Figure 6.C

20 shows that the peeling force is not constant during a peeling test on a patterned substrate. As the

21 first attached length ahead from the peeling front is reduced, the peeling force tends to decrease

22 until the detachment front encounters a detached region. Then, the force increases again as the 23 crack is stopped and the tape undergoes elastic deformation until the next attached length starts 
1 to peel-off. The difference between minimum and maximum detachment force in this case

2 increases as the unbounded lengths $\alpha$ are increased.

\subsection{Substrate curvature}

4 In [14], the ability of the gecko's lamellar system to adapt to wavy surfaces was studied. 5 Specifically the influence of substrates characterized by a sinusoidal profile on the shear 6 adhesion strength was considered. Depending on the amplitude and the wavelength of the 7 considered surface profile, the hierarchical adhesive pad of gecko performs a full or a partial 8 contact with the substrate. When partial contact is achieved, the problem is similar to that 9 discussed in the previous section, but when tape-like structures of lamellae conform to the 10 curvature of the surface, the process zone load distribution differs from the flat surface case. A 11 radius of curvature for convex and concave surfaces can be simply introduced in the present 12 model by setting the initial coordinates of the tape nodes along an arc of a circle rather than a 13 line. The peeling angle in this case is the angle between the applied external load and the tangent

14 to the substrate surface at the peeling line, and the adhesive energy is set to constant on all points 15 of the model. When the length of the process zone is small with respect to the radius of curvature 16 of the surface (in our case approximately $1 \mathrm{~mm}$ ), this effect is negligible, but as it decreases and 17 becomes of comparable size, the peeling force is affected. Figure 7 shows the peeling force, 18 normalized with respect to the peeling force on a flat substrate with the same parameters, as a 19 function of the radius of curvature. For convex surfaces, the peeling force increases as the 20 curvature radius decreases, and the opposite happens for concave surfaces. Additionally, the 21 peeling angle changes as the detachment proceeds and the peeling force is not constant during 22 propagation of the peeling front, contrary to the case for a flat surface. This is illustrated in 23 Figure 7.C, where the force tends to increase for convex surfaces, and decrease for concave 
1 surfaces. When the contacts are split, the adhesive strength is the result of simultaneous

2 delamination of multiple tapes [25], as is the case for animal hairy attachments, and the decrease

3 in local angles at different hierarchical scales could help in optimizing the total peeling force.

4

5

6

7

\section{Influence of substrate deformation}

In the case of soft materials, substrate deformation can also affect the way the load is distributed in peeling tests. The inclusion of substrate deformation in the calculation of the peeling force is a complex problem, since implementing an elastic half-plane in the simulation with a sufficient precision, i.e. a sufficiently small discretization length, would lead to a very large number of degrees of freedom. Instead, we propose to introduce an elastic foundation of stiffness $K_{S}$ in both normal and tangential directions for the substrate nodes. The interface is therefore introduced in the numerical model as a set of interface and substrate bonds arranged in series. The local failure criterion is still applied to the interface bonds when the system is loaded, in the hypothesis that no failure events take place within the substrate. Figure 8 shows, for a uniform adhesive energy, the peeling force as a function of the ratio between the interface and the substrate stiffness. A more compliant substrate generates a higher peeling force, which tends to the theoretical value of Eq. (4) as the substrate becomes stiffer. This increase is the result of two positive mechanisms: first, the distribution of the load in the interface becomes wider, which reduces the load concentration in the vicinity of the peeling line; second, as a result of the substrate deformation, the local peeling angle, i.e. the angle between the applied external load and the deformed surface of the substrate decreases. This mechanism is also favourable for an increase in the detachment (1) force. A limit of this gain lies in the fact that the attached length of the tape might not be long 
enough to indefinitely extend the width of the distribution. In this case, the peeling force increase

2 is limited.

3 Notice that these results are in contrast with experimental observations in [32], but as the authørs

4 themselves point out therein, the measurements are largely affected by other characteristics of

5 the adopted soft substrates, such as surface chemistry, capillary adhesion, friction, etc., all of

6 which are not assumed to vary in our model.

7

\section{Discussion}

We have modelled different geometrical and elastic features of the substrate surface profile in order to describe the adhesive behaviour of real materials, where roughness can be observed at different scale levels. We have shown that when the size scale of asperities is small compared to the process zone, their effect on the peeling properties of the tape is limited. We treated this case by considering the roughness as a statistical distribution on the adhesive bond energy to failure, since this implies that the attached length of the film is large with respect to the substrate roughness pattern, and found that the detachment force is close to the one obtained theoretically taking the average of the distribution. As the size of asperities becomes larger with respect to the attached length of the film, the detached areas become comparable to the process zone, and a decrease in the detachment force is observed. This is consistent with theoretical [17] and experimental [33] [20] [22] data, in which the predicted/measured detachment force for a rough surface is considerably affected by partial bonding and the pull-off force decreases monotonically with increasing surface roughness. Above a certain roughness size scale, partial bonding is no longer an issue, as the tape can conform to the surface pattern. In agreement with theoretical results from [18], there is some variation in the detachment force depending on the 
1 type of curved substrate on which the tape must adhere. Simulations show that this is the result

2 of the local perturbation in the process zone due to the change of peeling angle in the zone ahead

3 of the detachment front. As delamination proceeds, the detachment force is no longer constant,

4 rather it increases or decreases depending on the criticality of the new distribution emerging from

5 the varying angle of the attached length. The latter observation is consistent with the increase in

6 detachment force obtained experimentally above a certain roughness in [19], since a non-

7 negligible fraction of tape-like contacts is likely to be subjected to a smaller peeling angle as

8 delamination proceeds, and therefore a larger peeling force during simultaneous detachment. All

9 of these results are modified by non-negligible substrate compliance, which we show to be

10 advantageous to adhesive properties in the case of a uniform surface. Further dedicated

11 experimental tests to validate these numerical results would be desirable in the future.

\section{Conclusions}

14 In conclusion, we have developed a numerical model to simulate the peeling of adhesive tape15 like structures that is focused on the adhesive interface between tape and substrate. The 16 numerical procedure has been validated with classical thin film peeling theoretical model, and

17 further studies on the interface and substrate properties have been proposed. It was shown that 18 modelling the interfacial zone where the adhesive bonds are loaded, referred to as the "process 19 zone" allows to capture some/effects not predicted by the theoretical model, such as an imperfect 20 bonding, the geometry of the substrate surface or substrate deformations within the process zone.

21 The presented model and simulation contribute in providing a better understanding of the 22 mechanisms and possible optimization of adhesive structures. 


\section{Acknowledgements}

2 N.M.P. is supported by the European Commission under the Graphene FET Flagship (WP14 3 "Polymer composites" No. 604391) and FET Proactive "Neurofibres" grant No. 732344. FB is 4 supported by "Neurofibres" grant No. 732344. This work was carried out within the COST 5 Action CA15216 "European Network of Bioadhesion Expertise: Fundamental Knowledge to 6 Inspire Advanced Bonding Technologies". Computational resources were provided by 7 hpc@polito (www.hpc.polito.it).

\section{Appendix}

10 Further details regarding the implementation of the numerical model are provided below. For the

11 tape elements linking two nodes $i$ and $j$ of the model, mechanical equilibrium is implemented

12 using co-rotational truss formulation [34]. The material and geometric local stiffness matrices are 13 given by:

$$
\begin{gathered}
\mathbf{K}_{\mathbf{T m}}=\frac{E b w}{\Delta x}\left[\begin{array}{cccc}
n_{1}{ }^{2} & n_{1} n_{2} & -n_{1}{ }^{2} & -n_{1} n_{2} \\
n_{1} n_{2} & n_{2}{ }^{2} & -n_{1} n_{1} & -n_{2}{ }^{2} \\
-n_{1}{ }^{2} & -n_{1} n_{2} & n_{1}{ }^{2} & n_{1} n_{2} \\
-n_{1} n_{2} & -n_{2}{ }^{2} & n_{1} n_{2} & n_{2}{ }^{2}
\end{array}\right] \\
\mathbf{K}_{\mathbf{T m}}=\frac{E b w}{\Delta x} \frac{\delta_{T}}{\Delta x+\delta_{T}}\left[\begin{array}{cccc}
1 & 0 & -1 & 0 \\
0 & 1 & 0 & -1 \\
-1 & 0 & 1 & 0 \\
0 & -1 & 0 & 1
\end{array}\right]
\end{gathered}
$$

15 where $E$ is the elastic modulus of a tape element, $b$ its thickness, $w$ its width, $\Delta x$ its length 16 (corresponding to the discretization length), $\delta_{T}$ its elongation and $n_{1}$ and $n_{2}$ the components of 17 the (deformed) tape element direction vector in 2-D space, i.e. 


$$
\begin{aligned}
& n_{1}=\frac{x_{j}+u_{j}-x_{i}-u_{i}}{\Delta x+\delta_{T}} \\
& n_{2}=\frac{y_{j}+v_{j}-y_{i}-v_{i}}{\Delta x+\delta_{T}}
\end{aligned}
$$

2

3 where $\mathbf{x}=\left[\begin{array}{ll}x & y\end{array}\right]$ is the coordinate vector of a node and $\mathbf{u}=\left[\begin{array}{ll}u & v\end{array}\right]$ its displacement after 4 deformation. Its contribution tothe internal force vector is:

$$
\mathbf{Q}_{\mathbf{T i}}=\frac{E b w}{\Delta x} \delta_{T}\left[\begin{array}{c}
n_{1} \\
n_{2} \\
-n_{1} \\
-n_{2}
\end{array}\right]
$$

6

7 On the other hand, the interface bonds act on one node each (i). For a linear elastic traction-

8 separation law, the contribution of an interface bond to the stiffness matrix is given by:

10

$$
\mathbf{K}_{\mathbf{I}}=K_{I} w \Delta x\left[\begin{array}{ll}
1 & 0 \\
0 & 1
\end{array}\right]
$$

11 where $k_{I}$ is the stiffness of an interface bond. Its contribution to the internal force vector thus

12 becomes:

13

$$
\mathbf{Q}_{\mathbf{I i}}=K_{I} w \Delta x \delta_{I}\left[\begin{array}{l}
u_{i} \\
v_{i}
\end{array}\right]
$$


2 The external force vector $\mathbf{Q}_{\mathbf{e}}$ contains the components of the external load acting on the system.

3 Once all contributions are assembled into the linear system, mechanical equilibrium is obtained 4 by updating the nodal displacement according to the following iterative scheme:

$$
\mathbf{u}+\left(\mathbf{K}_{\mathbf{T m}}+\mathbf{K}_{\mathbf{T g}}+\mathbf{K}_{\mathbf{I}}\right)^{-1}\left(\mathbf{Q}_{\mathbf{e}}-\mathbf{Q}_{\mathbf{T i}}-\mathbf{Q}_{\mathrm{Ii}}\right) \rightarrow \mathbf{u}
$$

6

7 The 2-norm of the residual $\left\|\mathbf{Q}_{\mathbf{e}}-\mathbf{Q}_{\mathrm{Ti}}-\mathbf{Q}_{\mathrm{Ii}}\right\|$ is used as convergence criterion.
8

10

[1] Qiang, C.; Pugno, N. M., "Bio-mimetic mechanisms of natural hierarchical materials: a review.," Journal of the Mechanical Behavior of Biomedical Materials, vol. 19, pp. 3-33, 2013.

[2] Xia, F.; Jiang, L., "Bio-inspired, smart, multiscale interfacial materials," Advanced materials, vol. 20, no. 15, pp. $2842-2858,2008$.

[3] Youngblood, J. P.; Sottos, N. R., "Bioinspired materials for self-cleaning and self-healing.," Mrs Bulletin, vol. 33, no. 08, pp. 732-741, 2008.

[4] C. Sanchez, H. Arribart and M. Giraud Guille, "Biomimetism and bioinspiration as tools for the design of innovative materials and systems," Nature Materials, vol. 4, pp. 277-288, 2005.

[5] Varenberg, M.; Pugno, N.M.; Gorb, S.N., "Spatulate structures in biological fibrillar adhesion," Soft Matter, vol. 6, no. 14, pp. 3269-3272, 2010.

[6] K. Autumn, A. Dittmore, D. Santos, M. Spenko and M. Cutkosky, "Frictional adhesion: a new angle on gecko attàchment," The Journal of Experimental Biology, vol. 209, pp. 3569-3579, 2006.

[7] Pesika, N.S.; Tian, Y.; Zhao, B.; Rosenberg, K.; Zeng, H.; McGuiggan, P.; Autumn, K.; Israelachvili, J.N., "Peel-zone model of tape peeling based on the gecko adhesive system.," The Journal of Adhesion, vol. 83, no. 4, pp. 383-401, 2007. 
[8] Tian, Y.; Pesika, N.; Zeng, H.; Israelachvili, J., "Adhesion and friction in gecko toe attachment and detachment," Proceedings of the National Academy of Sciences, vol. 103, no. 51, pp. 19320-19325, 2006.

[9] R. S. Rivlin, "The effective work of adhesion," Paint Technology, vol. IX, no. 106, p. 2611-2614, 1944.

[10] K. Kendall, "Thin-film peeling-the elastic term," Journal of Physics D: Applied Physics, vol. 8, no. 13, p. 1449, 1975.

[11] Autumn, K., "Evidence for van der Waals adhesion in gecko setae," Proceedings of the National Academy of Sciences, vol. 99, no. 19, pp. 12252-12256, 2002.

[12] Federle, W.; Riehle, M.; Curtis, A. S. G.; Full, R. J., "An integrative study of insect adhesion: mechanics and wet adhesion of pretarsal pads in ants," Integrative and Comparative Biology, vol. 42, no. 6, pp. 1100-1106, 2002.

[13] Zhou, Y.; Robinson, A.; Steiner, U.; Federle, W., "Insect adhesion on rough surfaces: analysis of adhesive contact of smooth and hairy pads on transparent microstructured substrates," Journal of The Royal Society Interface, vol. 11, no. 98, 2014.

[14] Gillies, A.G.; Henry, A.; Lin, H.; Ren, A.; Shiuan, K.; Fearing, R.S.; Full, R. J. , “Gecko toe and lamellar shear adhesion on macroscopic, engineered rough surfáces," Journal of Experimental Biology, vol. 217, no. 2, pp. 283-289, 2014.

[15] Niewiarowski, P. H.; Stark, A.Y.; Dhinojwala, A., "Sticking to the story: outstanding challenges in gecko-inspired adhesives.," Journal of Experimental Biology, vol. 219, no. 7, pp. 912-919, 2016.

[16] B. Persson, "On the mechanism of adhesion in biological systems," The Journal of Chemical Physics, vol. 118 , no. 16, p. 7614-7621, 2003.

[17] B. N. J. Persson and S. Gorb, "The effect of surface roughness on the adhesion of elastic plates with application to biological systems," The Journal of Chemical Physics, vol. 119, p. 11437, 2003.

[18] Z. L. Peng and S. H. Chen, "Effects of surface roughness and film thickness on the adhesion of a bioinspired nanofilm," Physical Review E, vol. 83, no. 5, p. 051915, 2011.

[19] G. Huber, S. Gorb, N. Hosoda, R. Spolenak and A. Arzt, "Influence of surface roughness on gecko adhesion," Acta Biomaterialia, vol. 3, no. 4, pp. 607-610, 2007.

[20] N. Cañas, M. Kamperman, B. Völker, E. Kroner, R. McMeeking and E. Arzt, "Effect of nano- and micro-roughness on adhesion of bioinspired micropatterned surfaces," Acta Biomaterialia, vol. 8, pp. 282-288, 2012.

[21] P. Lin, S. Vajpayee, A. Jagota, C. Hui and Y. S., "Mechanically tunable dry adhesive from wrinkled elastomers," Soft Matter, vol. 4, pp. 1830-1835, 2008. 
[22] A. Jagota and C. Hui, "Adhesion, friction, and compliance of bio-mimetic and bio-inspired structured interfaces," Materials Science and Engineering R, vol. 72, p. 253-292, 2011.

[23] Rivlin, R. S.; Thomas, A. G., "Rupture of rubber. I. Characteristic energy for tearing," Collected Papers of RS Rivlin. Springer New York, pp. 2615-2642., 1997.

[24] D. H. Kaelble, "Theory and analysis of peel adhesion: bond stresses and distributions," Transactions of The Society of Rheology, vol. 4, no. 1, pp. 45-73, 1960.

[25] N. M. Pugno, "The theory of multiple peeling," International journal of fracture, vol. 171, no. 2, pp. 185-193, 2011.

[26] H. L. Cox, "The elasticity and strength of paper and other fibrous materials," British journal of applied physics, vol. 3, no. 3, p. 72, 1952.

[27] Z. Peng and S. Chen, "Effect of bending stiffness on the peeling behavior of an elastic thin film on a rigid substrate," Physical Review E, vol. 91, p. 042401, 2015.

[28] G. I. Barenblatt, "The mathematical theory of equilibrium cracks in brittle fracture.," Advances in applied mechanics, vol. 7, pp. 55-129, 1962.

[29] K. Atkinson, An introduction to numerical analysis, John Wiley \& Sons, 2008.

[30] J. Bullock and W. Federle, "Division of labour and sex differences between fibrillar, tarsal adhesive pads in beetles: effective elastic modulus and attachment performance," Journal of Experimental Biology, vol. 212, no. 12, pp. 1876-1888, 2009.

[31] P. Henrik, J. Michels and S. Gorb, "Evidence for a material gradient in the adhesive tarsal setae of the ladybird beetle Coccinella septempunctata," Nature communications, vol. 4, p. 1661, 2013.

[32] M. R. Klittich, M. Wilson, C. Bernard, R. Rodrigo, A. Keith, P. H. Niewiarowski and A. Dhinojwala, "Influence of substrate modulus on gecko adhesion," Scientific Reports, vol. 7, p. 43647, 2017.

[33] K. Fuller and D. Tabor, "The effect of surface roughness on the adhesion of elastic solids," Proc Roy Soc London A, vol. 345, p. 327, 1975.

[34] L. L. Yaw, “3D Co-rotational Truss Formulation," 2011. [Online]. Available: https://gab.wallawalla.edu/ louie.yaw/Co-rotational_docs/3Dcorot_truss.pdf.

[35] Autumn, K.; Dittmore, A.; Santos, D.; Spenko, M.; Cutkosky, M. , "Frictional adhesion: a new angle on gecko attachment," Journal of Experimental Biology, vol. 209, no. 18, pp. 3569-3579, 2006. 
A

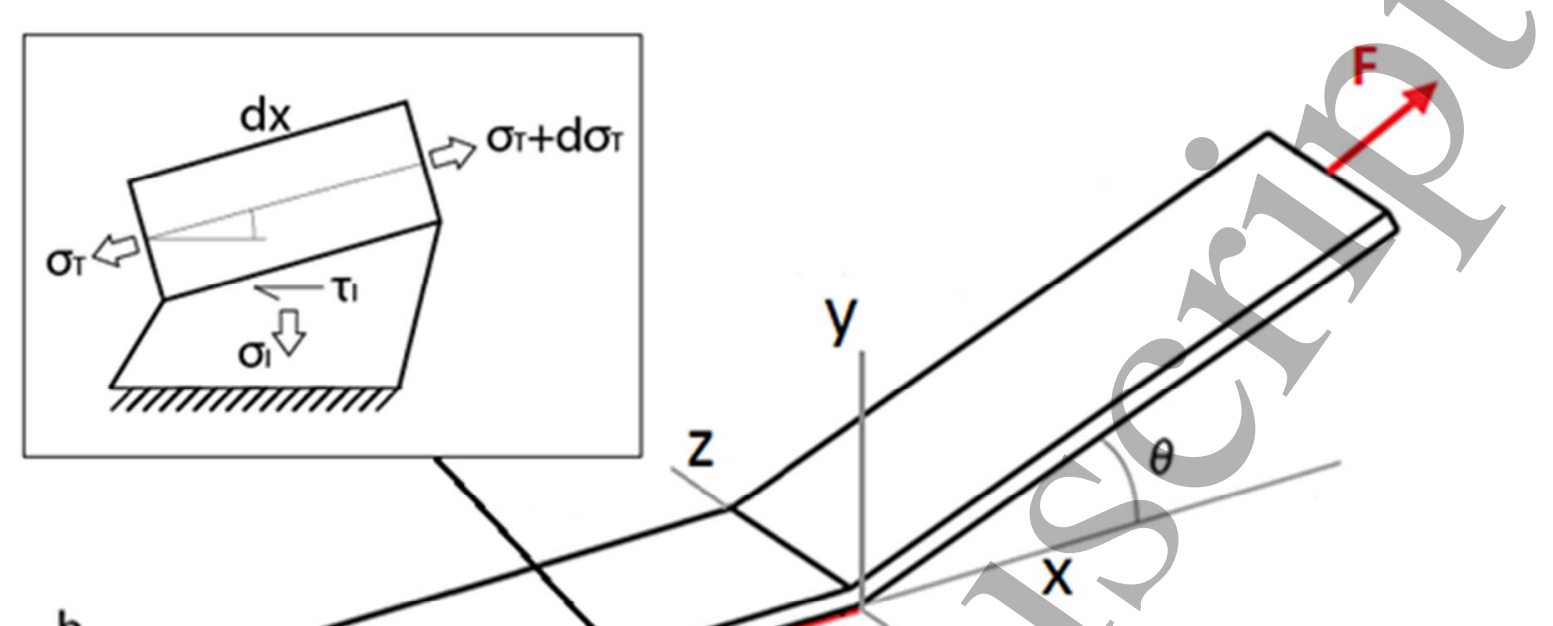

1
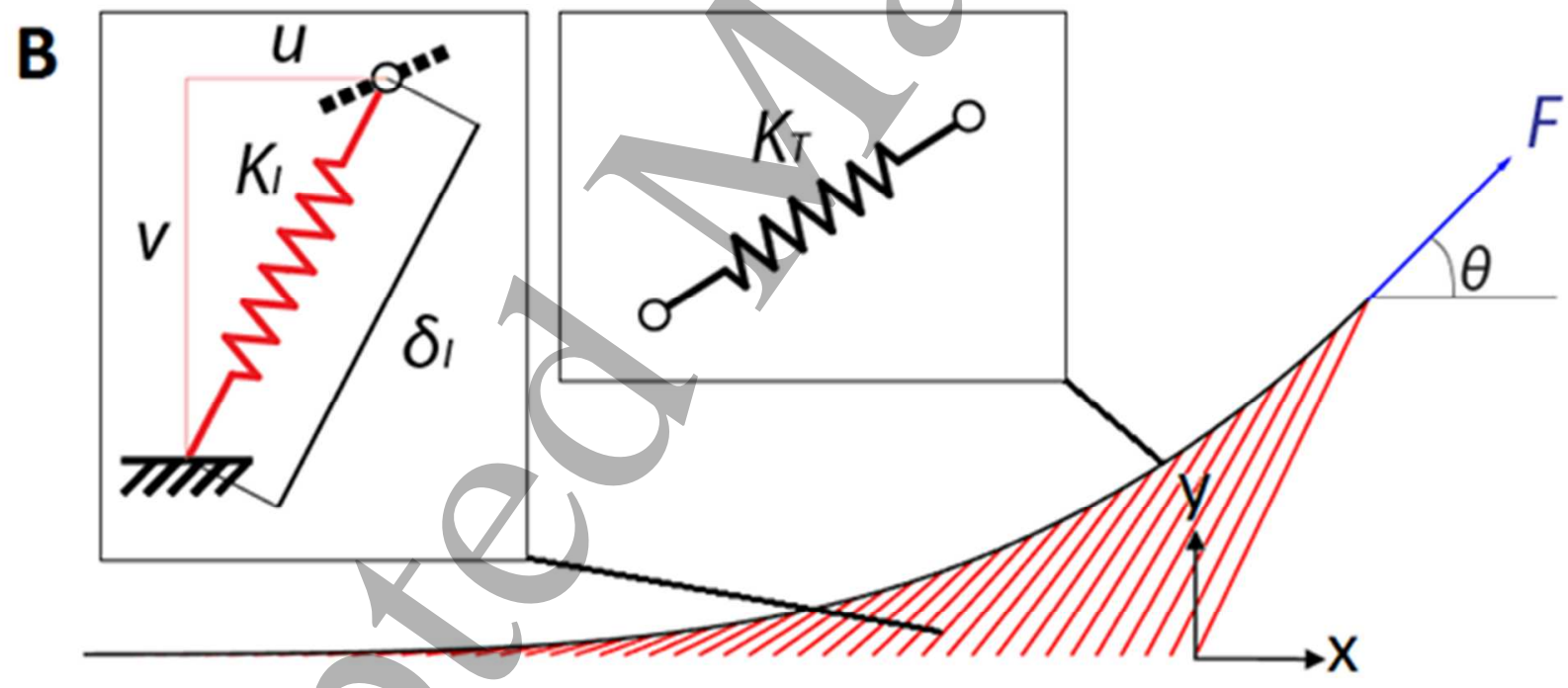

2 Figure 1:A. Schematic representation of the geometry of the considered tape peeling problem

3 and (inset) of the stresses acting on an elementary unit of the interface. B. Corresponding 4 discretization in the numerical model using interface and tape spring elements of stiffnesses $K_{I}$ 5 and $K_{T}$, respectively. 

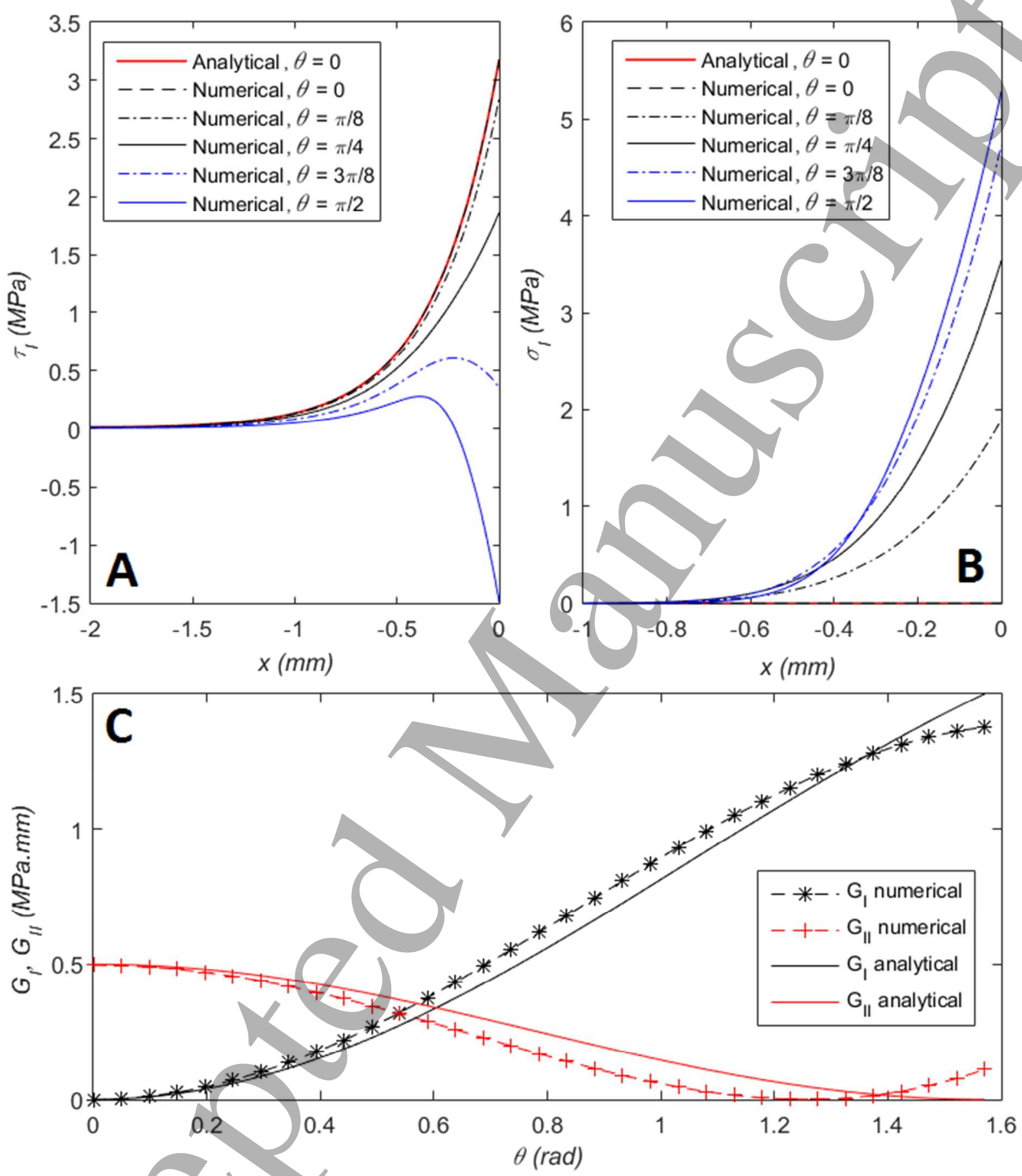

1

2 Figure 2:A. Shear and B. Normal stress distributions at the interface along the process zone for

3 different peeling angles. C. Mode I and mode II strain energy release rate as a function of the 4 peeling angle. 


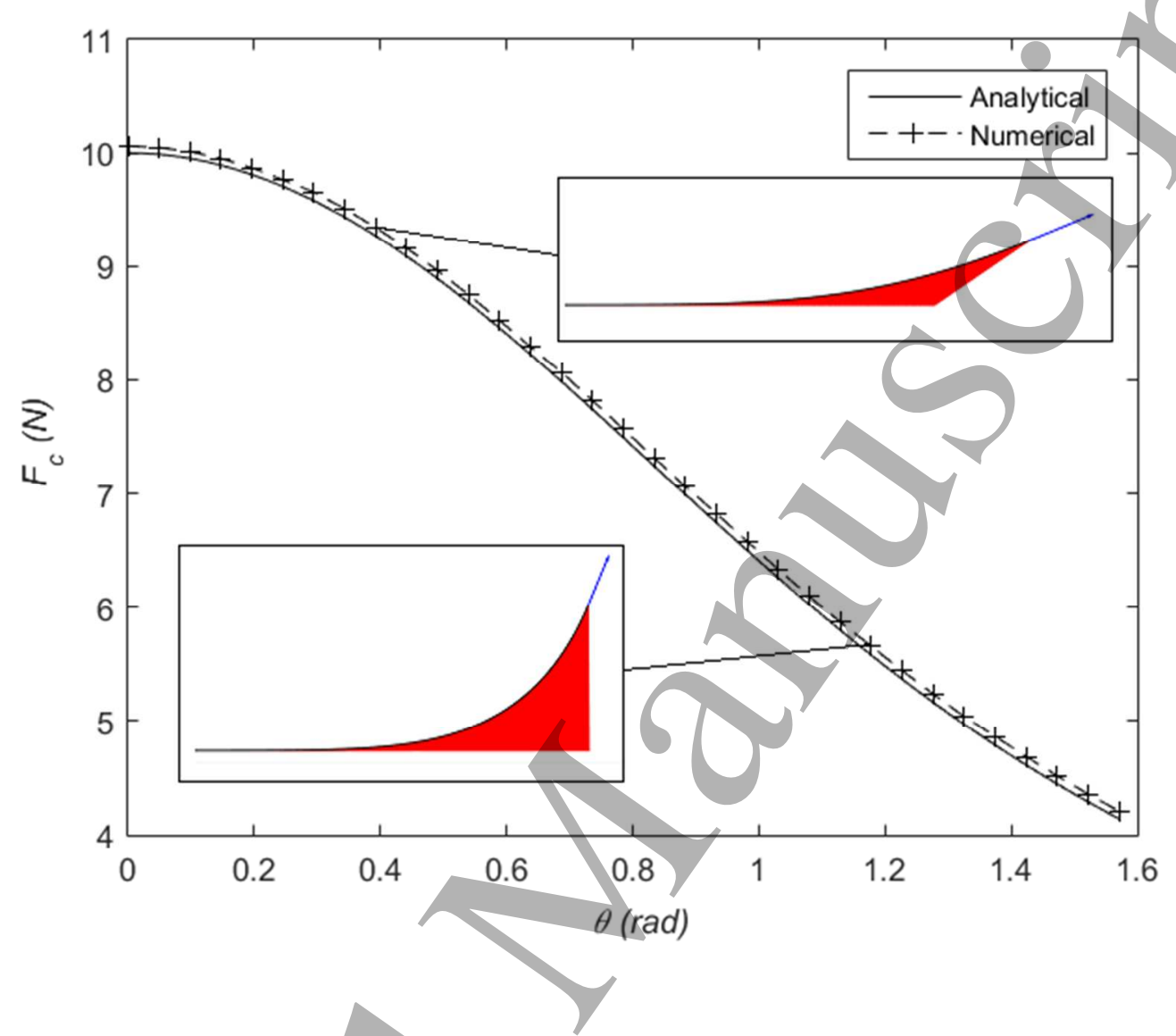

3 Figure 3 : Peeling force vs. peeling angle with the present numerical model and the theoretical 4 result, Inset: interface deformation states at two different peeling angles. 

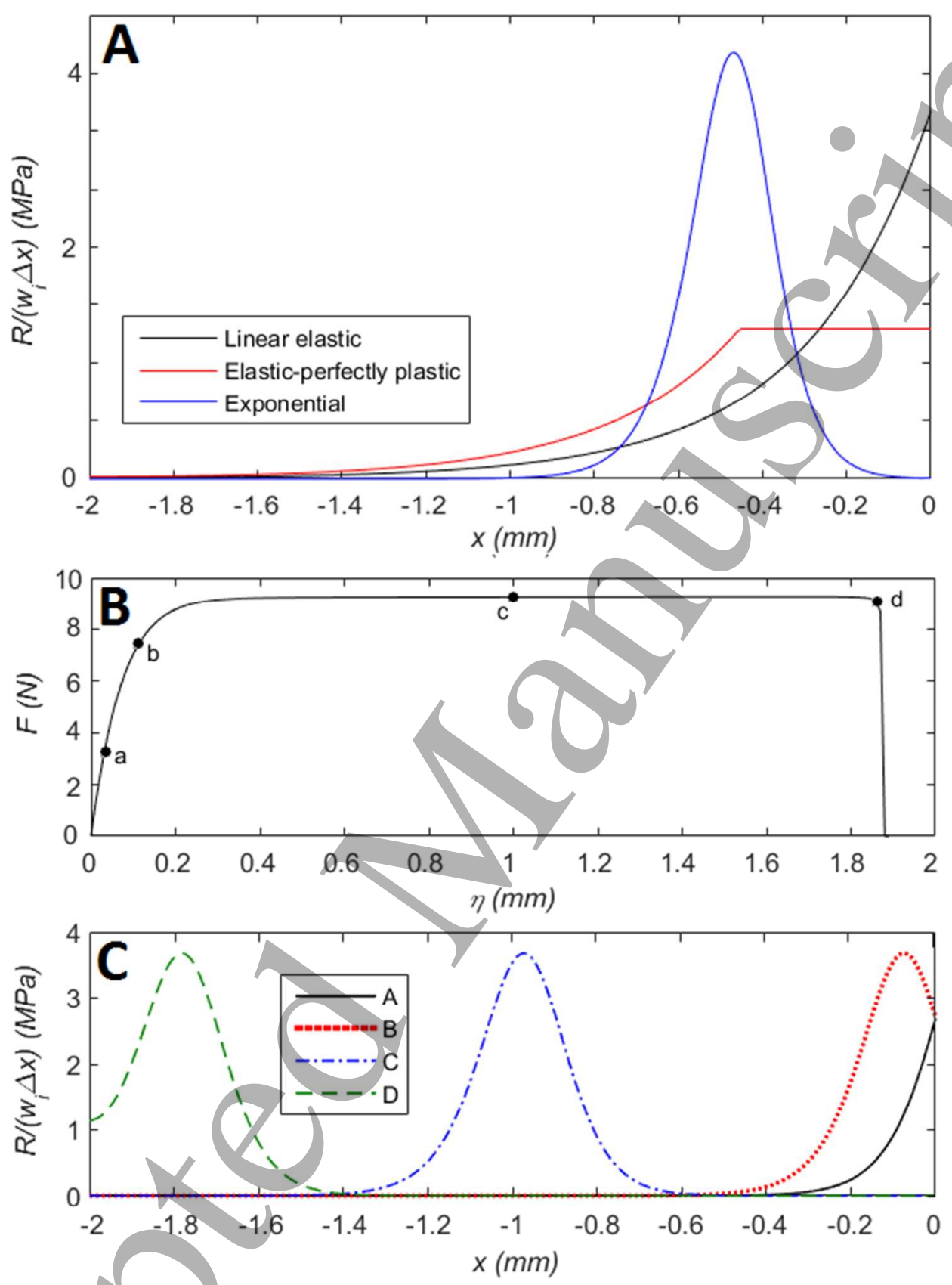

1

2 Figure 4: A. Interface bond axial stress along the process zone for different traction-separation 3 laws when the peeling force is reached (for $\theta=\pi / 8$ ). B. Resulting force-displacement 4 relationship in the peeling test for an exponential traction-separation law. C. Propagation of the 5 peeling front corresponding to points $a, b, c, d$, visualized using the interface bond axial stress 6 distribution. 


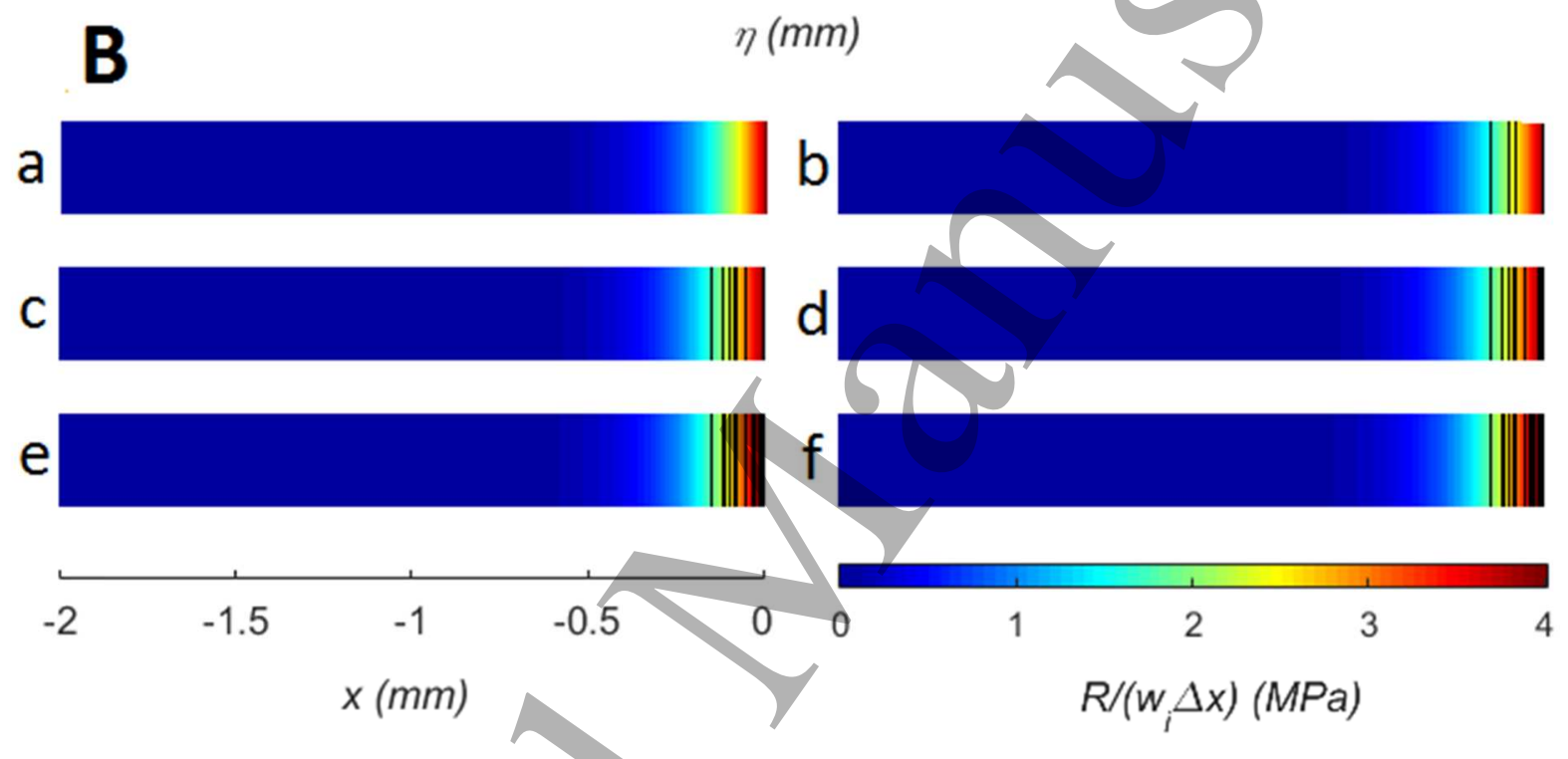

2 Figure 5 : Force $F$ vs. displacement $\eta$ during peeling of a tape from a substrate with nonzero

3 surface roughness for two different peeling angles and roughness distributions (compare to Fig.

44 for a smooth surface). B. Corresponding propagation of the peeling front in the early stages

5 of detachment at points a to falong the Fvs. $\eta$ curve. The color scale represents the interfacial

6 load distributions. The areas where local detachments occur are represented in black.

A

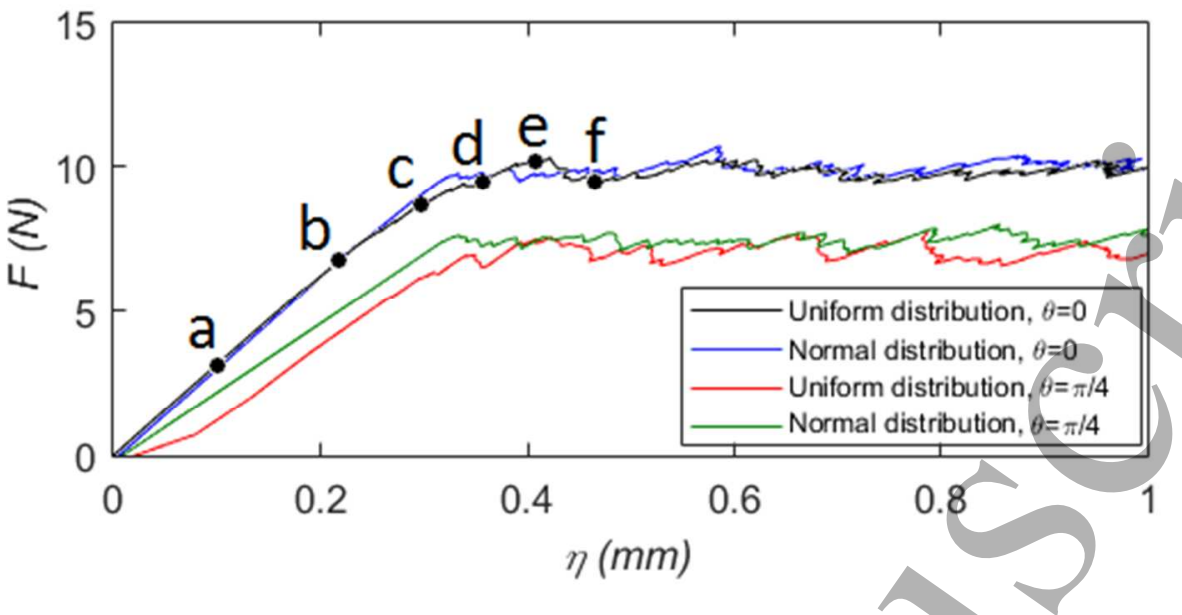


A

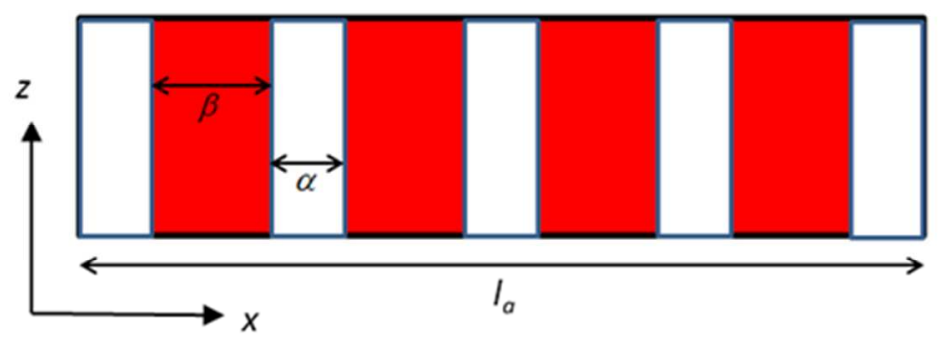

B
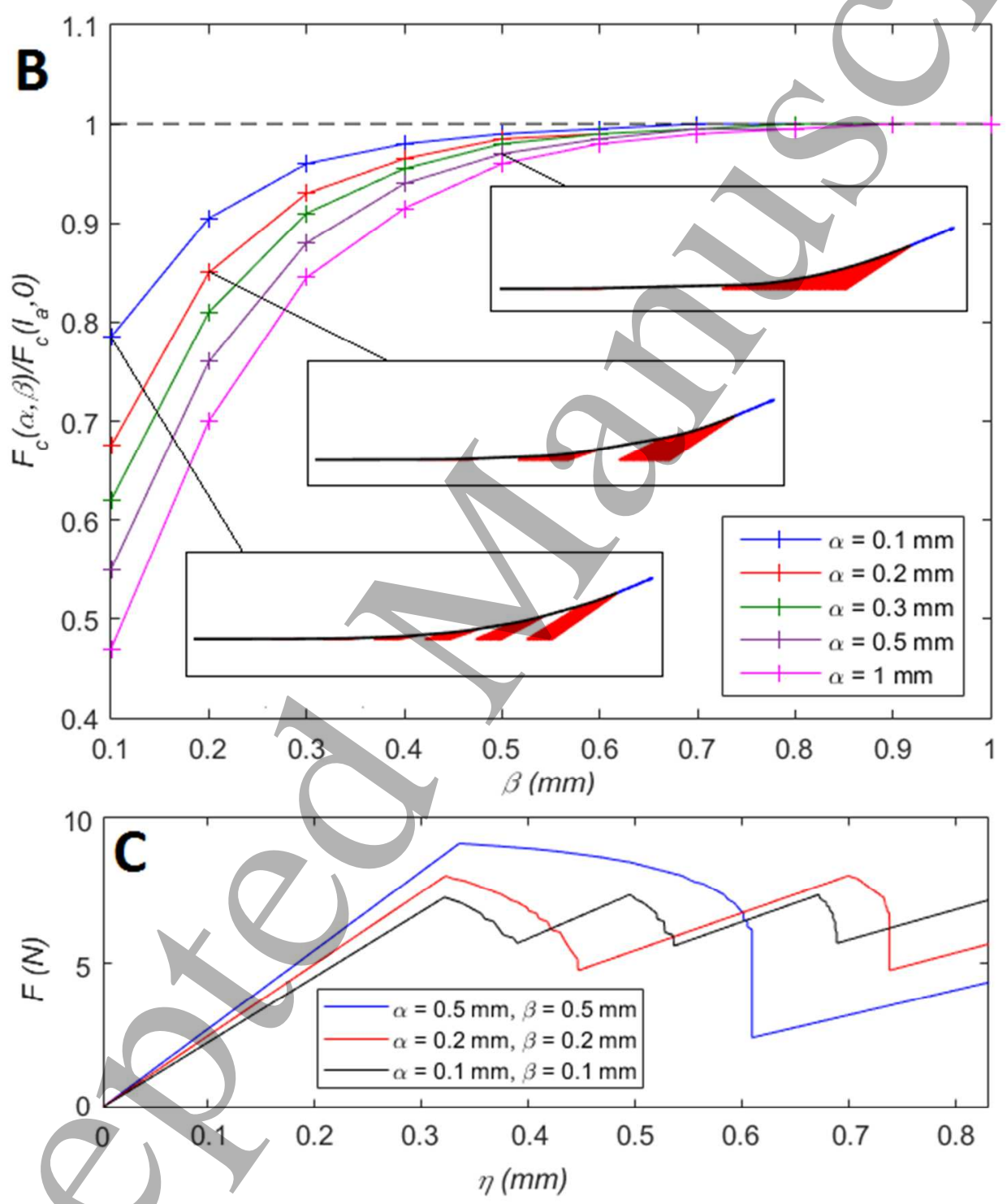

1

2 Figure 6:A. Schematic of the periodic patterned surface with alternating non-contacting lengths $3 \alpha$ and lengths in contact $\beta$. B. Normalized peeling force vs. $\beta$ for various $\alpha$ values. Inset: 
1 corresponding deformation states. C. Corresponding force $F v$ s. displacement $\eta$ during peeling 2 test.

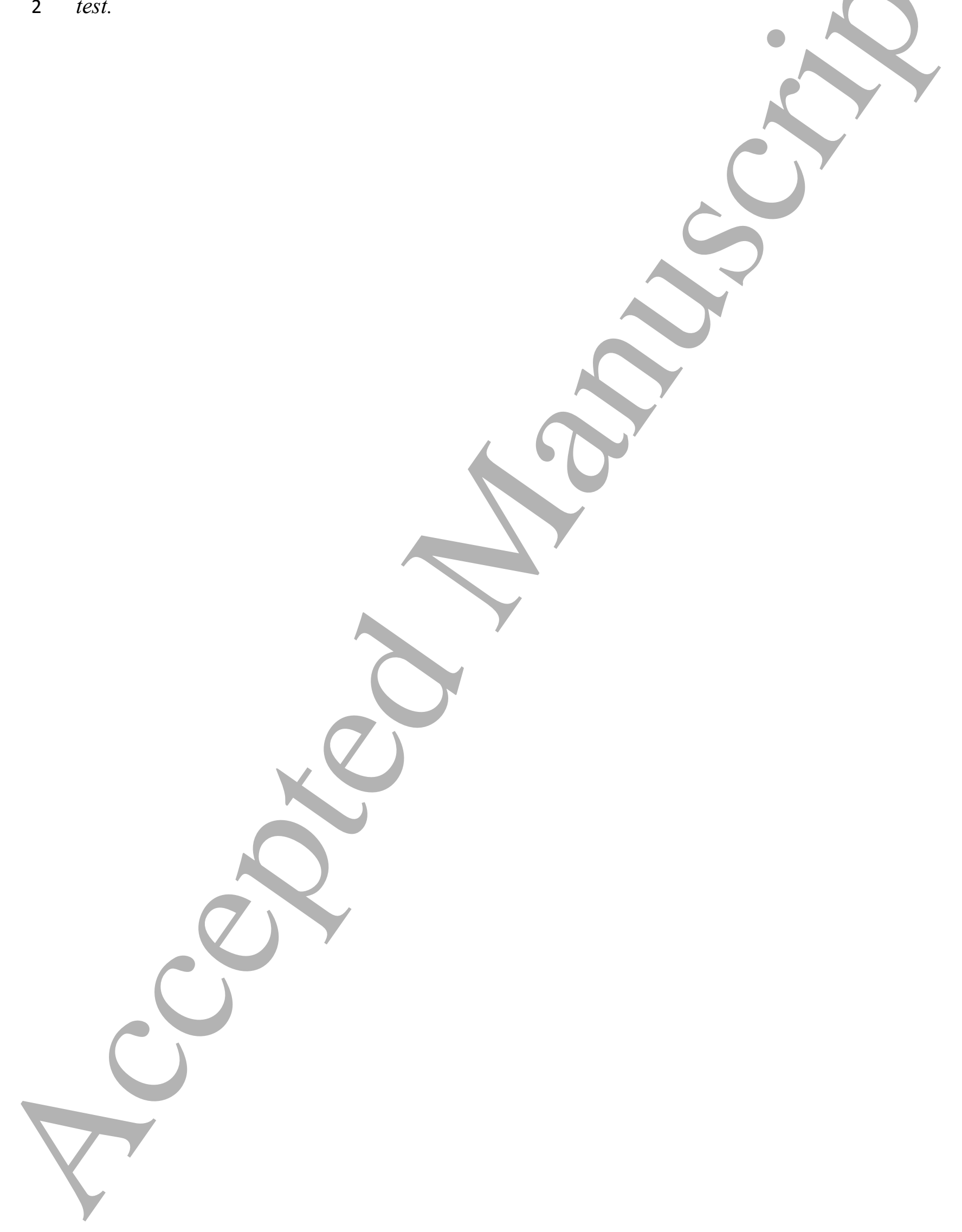



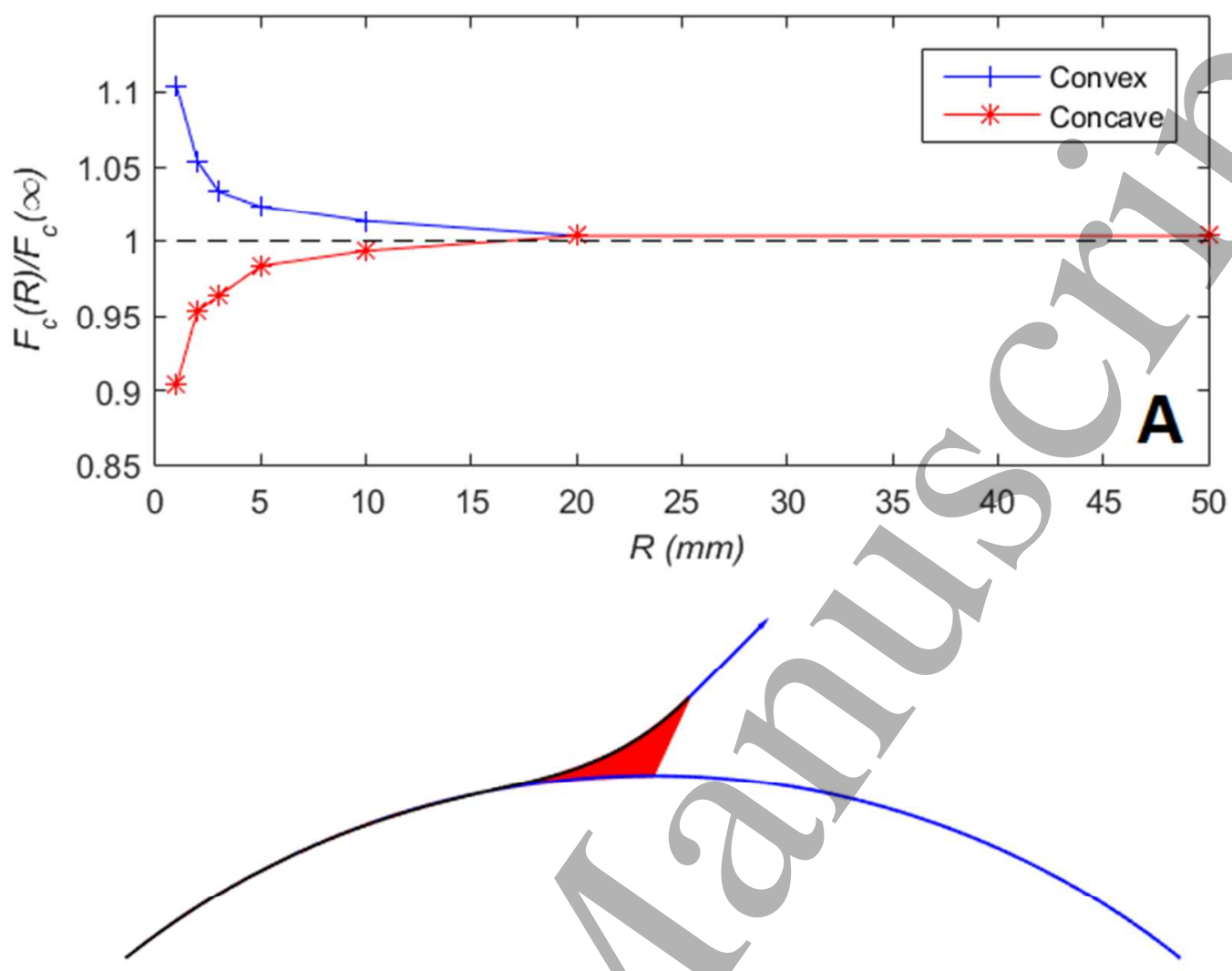

2 Figure $7:$. Normalized peeling force as a function of the radius of curvature of the substrate

3 for concave and convex surfaces. B. Corresponding deformation states. C. Corresponding force $4 \quad F$ vs. displacement $\eta$ during peeling test. 


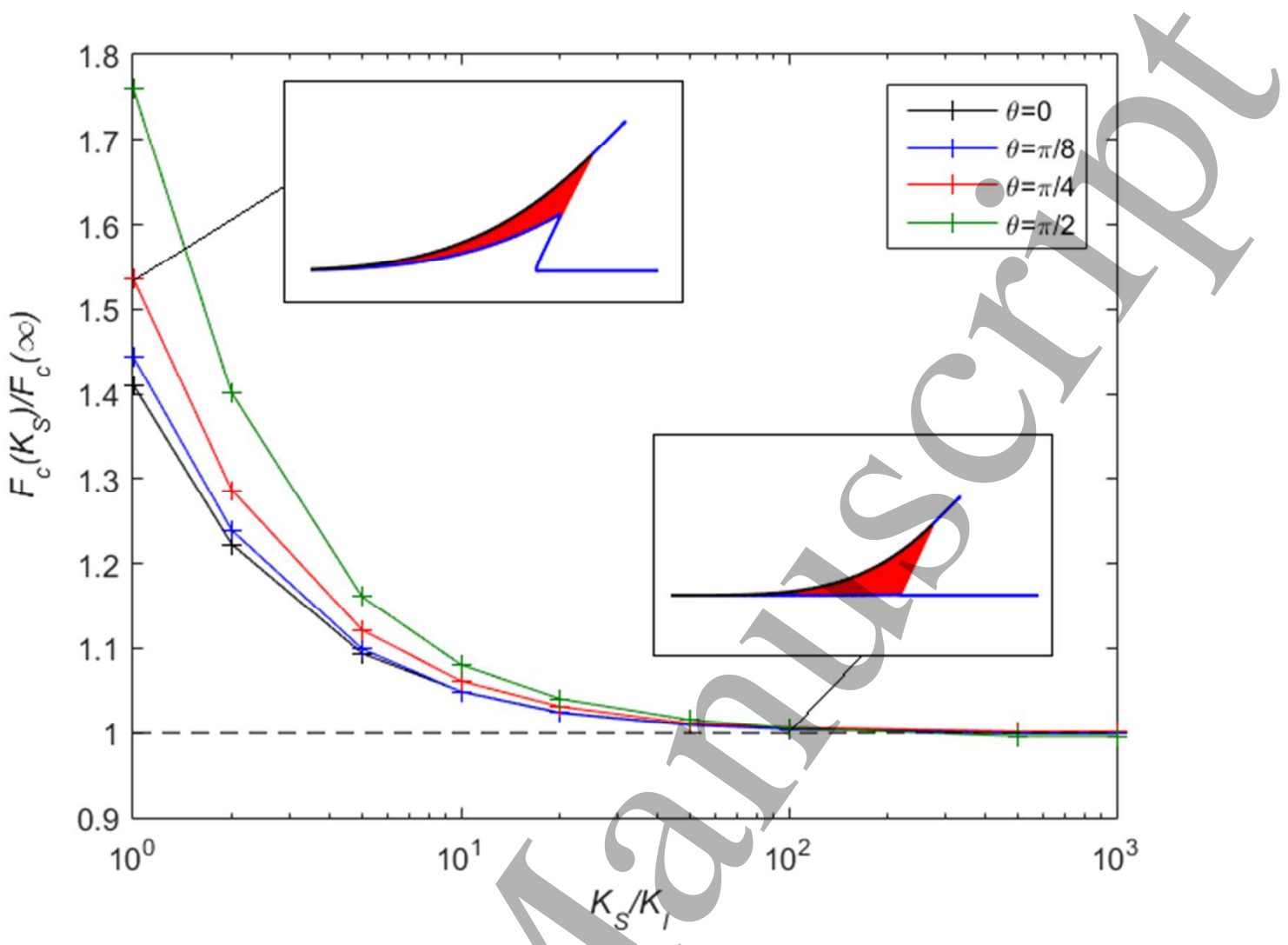

1

2 Figure 8: Normalized peeling force as a function of the substrate compliance for various 3 peeling angles. Inset: deformation states for various deformed substrate.

4 